\title{
Protestforschung mit politischem Auftrag?
}

\author{
Simon Teune/Peter Ullrich
}

Die Forschung zu Protesten und sozialen Bewegungen ist eine politische Wissenschaft: Sie ist zum Ersten ein Teilgebiet der Politikwissenschaft und der politischen Soziologie, weil sie sich mit ihrem Fokus auf einen Akteurstyp ausrichtet - soziale Bewegungen, mobilisierte Zivilgesellschaft und Aktivist"innen - und mit Fragen von Macht und der Herstellung verbindlicher Entscheidungen befasst; ihr Gegenstand ist also politisch. Sie ist es auch, zweitens, weil ein nicht unerheblicher Teil der Forschenden diesen Gegenstand aufgrund eigenen Engagements wählt und zumindest Teilen des Forschungsfeldes mit Sympathien gegenübersteht. Am deutlichsten wird das bei denen, die die eigene Forschung explizit in den Dienst der Bewegung(en) stellen, sei es als kritisches Reflexions- oder als strategisches Handlungswissen. Protestforschung ist, drittens, politisch, weil sie - etwa in der Medienfigur der Protestforscher*in - zur gesellschaftlichen Konstruktion ibres Gegenstands beiträgt.

Die akademische Würdigung von Protest hat auch zu seiner Normalisierung als akzeptierter Form politischer Partizipation beigetragen. Viertens ist Protestforschung politisch, weil ihr Tun immer wieder als Politikum empfunden wird. Protestforscher*innen sind immer konfrontiert mit Erwartungen und Einflüssen unterschiedlicher gesellschaftlicher Felder (Wissenschaft, Politik, Protestierende, Medien u.a.), die teilweise diametral entgegengesetzt sind und sich gelegentlich auch konflikthaft entladen: Auf der einen Seite vermuten wissenschaftspolitisch Verantwortliche, dass mit der Förderung der Protestforschung die Revolution finanziert werde. ${ }^{1}$ Auf der anderen Seite lehnen auch manche Aktivist*innen Protestforschung mit der Begründung ab, sie diene als Produktion von Herrschaftswissen der Einhegung von Protest. Protestforscher*innen sind damit nolens volens Teil einer politischen Auseinandersetzung. Daraus ergibt sich die Notwendigkeit zur Reflexion und Positionierung.

Während die Diskussion über die politischen Implikationen der Protestforschung das Feld schon immer begleitet hat, stellen sich in den letzten Jahren neue Fragen. Das Bekanntwerden des Nationalsozialistischen Untergrunds (NSU) und die Konjunktur völkischer Proteste wie etwa bei Pegida hat ein neues Interesse an sozialen Bewegungen von rechts geweckt. Schon vorher hatte sich mit dem Extremismusparadigma in Behörden und Ministerien die Vorstellung durchgesetzt, dass zwar der Rechtsextremismus gut erforscht sei, es aber an Erkenntnissen zum „Linksextremismus" fehle. Mit diesen Entwicklungen hat sich der Blick auf Protestforschung verändert und es entstanden neue Institutionen, die das Feld mit politischem Auftrag in den Blick nehmen.

\section{Forschungsförderung in versicherheitlich- ter Perspektive}

Nachdem die zweifelhafte Rolle der Geheimdienste in der Entstehung und ausbleibenden Aufklärung des NSU offenkundig wurde, entschlossen sich die Länder Thüringen, Niedersachsen und Brandenburg, die Arbeit des Verfassungsschutzes durch neue sozialwissenschaftliche Einrichtungen zu flankieren. Das „Institut für Demokratie und Zivilgesellschaft" in Jena, die „Emil Julius Gumbel Forschungs- 
stelle Antisemitismus und Rechtsextremismus“ in Potsdam und die „Forschungs- und Dokumentationsstelle zur Analyse politischer und religiöser Extremismen in Niedersachsen (FoDEx) “ im Göttinger Institut für Demokratieforschung sollten mit überprüfbaren Methoden praktisches Wissen generieren. $^{2}$

Es ist kaum zu bezweifeln, dass sozialwissenschaftliche Analysen wie auch zivilgesellschaftliche Rechercheinitiativen mehr zur Aufklärung von rechter Alltagsgewalt und rechtem Terror leisten als die Verfassungsschutzbehörden. Diese neuen Einrichtungen bieten eine Chance, eine eingreifende Forschung in öffentlicher Verantwortung neu zu denken. Ob aber diese Chance genutzt werden kann, ist davon abhängig, welches Wissen von den Geldgebern erwartet wird und welche Entwicklungen in den Blick genommen werden.

Mit der staatlichen Beauftragung geht auch eine Prägung durch außerwissenschaftliche Ansprüche und Erkenntnisinteressen einher, die für die Protest- und Bewegungsforschung unmittelbare Konsequenzen hat. Zum einen verändert der politische Auftrag die Rolle wissenschaftlicher Akteure in ihrem Forschungsfeld. Zum anderen drückt das im politischen Diskurs hegemoniale Extremismusparadigma, also die Annahme, dass die demokratische Gesellschaft von politischen Randgruppen bedroht sei, der Arbeit der Einrichtungen ihren Stempel auf. Historisch als Rechtfertigung für Berufsverbote und Radikalenerlass entstanden und in der Wissenschaft wegen seiner analytischen Unschärfe kritisch diskutiert (Forum für kritische Rechtsextremismusforschung 2011; Ackermann et al. 2015), erweist es sich heute vor allem durch die implizite Gleichsetzung von so unterschiedlichen Phänomenen wie dschihadistischen Anschlägen, rassistischen Morden und Konfrontationsgewalt bei Demonstrationen als problematisch. Die Erwartung, dass die neu entstandenen Einrichtungen „demokratiefeindliche“, „verfassungsfeindliche“ oder „,extremistische“ Tendenzen in den Blick nehmen, trägt nun dazu bei, dass eine versicherheitlichte Perspektive auch in die Forschung zu Protesten und sozialen Bewegungen Einzug hält.
In allen drei Institutionen sind Proteste ein Untersuchungsgegenstand. Allerdings zeichnen sich unterschiedliche Zugänge ab. Das Göttinger Institut für Demokratieforschung, ein wichtiges Zentrum der institutionell insgesamt nur schwach verankerten Protestforschung in der Bundesrepublik (Rucht 2011), nimmt in der ersten Publikation der Göttinger Forschungsund Dokumentationsstelle die G20-Proteste, Salafismus und eine Demonstration der Identitären in den Blick. In Potsdam werden Protestereignisse neonazistischer und völkischer Akteure dokumentiert und eingeordnet. In Jena entsteht eine regionale Protestereignisanalyse.

Inwiefern sich in den neuen Instituten mit der Finanzierung aus Landesmitteln auch deren demokratie- und sicherheitspolitische Logik auf das Forschungsfeld überträgt hängt indes davon ab, welche Zielvorstellungen im jeweiligen institutionellen und landespolitischen Kontext handlungsleitend sind: In Potsdam, wo SPD und Linke regieren, liegt der Schwerpunkt im Sinne des Trägers, des Moses Mendelssohn Zentrums, auf Antisemitismus und der extremen Rechten sowie den Bedingungen zivilgesellschaftlicher Resilienz. Die Jenaer Forscher*innen konzentrieren sich - ebenfalls im Sinne der Trägerin, der Amadeu Antonio Stiftung und mit Förderung durch die rotrot-grüne Landesregierung - auf die Analyse von Diskriminierung, Hasskriminalität und Hassgruppen im Sinne des Modells der „vorurteilsgeleiteten Radikalisierung" (Quent 2017).

Während die Vorgaben in diesen beiden Fällen eher lose formuliert sind, kommt bei der Göttinger Einrichtung die landespolitische Prägekraft in ihren Widersprüchen deutlicher zum Ausdruck: Nachdem der rot-grüne Koalitionsvertrag von 2013 „die öffentliche Bewertung verfassungsfeindlicher Bestrebungen " ${ }^{3}$ durch eine wissenschaftliche Stelle vorsah und die Grünen diesen Schritt mit der Forderung einer Abkehr vom Konzept des Extremismus verbanden, bezeichnete SPD-Innenminister Pistorius die Einrichtung bei ihrer öffentlichen Vorstellung als „wichtige[n] Schritt beim Kampf gegen Extremismus in unserem Land“" . Während die Forschungsstelle zum damaligen 
Zeitpunkt unter dem Namen „Dokumentationsstelle zur Analyse und Bewertung von Demokratiefeindlichkeit und politisch motivierter Gewaltbereitschaft in Niedersachsen " firmierte, trägt sie heute das Extremismusparadigma im Namen. Ihre Arbeit gliedert sich entlang der Themen „Rechtsextremismus, religiöser Extremismus und linke Militanz" (Trittel et al. 2017: 2), also mehr oder weniger entlang der „Phänomenbereiche” des Verfassungsschutzes, des polizeilichen Staatsschutzes und staatlicher Interventionsprogramme. Diese Ausrichtung stellen die in Göttingen Forschenden in ihrer ersten Veröffentlichung selbst in Frage. Äußerst kritisch setzen sie sich mit den affirmativen Selbstbeschreibungen der „streitbaren Demokratie" und insbesondere mit dem Konzept des Extremismus auseinander und betonen deren analytische Defizite (Riedl/Micus 2017; Trittel et al. 2017).

Dass sich die Forschungs- und Dokumentationsstelle zugleich der Aufarbeitung des Archivs des Landesamts für Verfassungsschutz widmet, dass sie gemeinsame Veranstaltungen mit diesem plant und dass am Göttinger Institut zudem eine „Bundesfachstelle linke Militanz" aus Mitteln des Bundesfamilienministeriums eingerichtet wurde, verstärkt dagegen den Eindruck, dass hier Protestforschung und staatliche Kontrollperspektive amalgamiert werden. Da darüber hinaus FoDEx wie auch Bundesfachstelle für Außenstehende nur schwer zu durchschauen sind, ${ }^{6}$ ist das Göttinger Institut schnell zur Zielscheibe von harscher Kritik geworden - im akademischen wie auch im politischen Feld. ${ }^{7}$

\section{Resonanzen im Feld}

Als Teams aus Dresden, Göttingen und dem Institut für Protest- und Bewegungsforschung (ipb) bei den Pegida-Demonstrationen Teilnehmer"innen befragen wollten, schlug ihnen deutliche Ablehnung entgegen, wohl weil sie als Teil der Deutungseliten und damit als politische Gegner"innen wahrgenommen wurden. Die Erfahrung dieser „Protestforschung am Limit“ (Daphi et al. 2015) hat Debatten über Forschungsethik und Grenzen der Methode der Demonstrationsbefragung ausgelöst (Reuband 2015; Teune/Ullrich 2015). Seit der Einrichtung der Forschungs- und Dokumentationsstellen haben sich die Anfeindungen aus dem Feld nochmals verschärft. Sowohl das Göttinger Institut für Demokratieforschung als auch das Institut für Demokratie und Zivilgesellschaft sehen sich einerseits heftigen Angriffen seitens der AfD ausgesetzt. Die institutionellen Geldgeber und Kooperationspartner der neuen Einrichtung in Göttingen sowie der Mangel an Informationen über ihre Tätigkeit haben andererseits auch dazu beigetragen, dass die Spekulationen in der linken Szene ins Kraut schießen - bis hin zur Einordnung der FoDEx als „VS-Projekt”. Im November 2017 leerten Aktivist"innen in Trenchcoat und Schlapphut säckeweise Papierschnipsel vor dem Institut aus, um zu skandalisieren, dass die Forschungsstelle „mit und für den Verfassungsschutz $^{\text {"8 }}$ arbeite. In einzelnen Treffen der linken Szene und Wohngemeinschaften wurden Mitarbeiter*innen der Göttinger Einrichtungen zu unerwünschten Personen erklärt.

Diese Zuspitzung hat schon jetzt spürbare Auswirkungen auf das Verhältnis zwischen der Protestforschung und ihrem Forschungsfeld, die in ihrer ganzen Tragweite noch nicht absehbar sind. Die Annahme, die Protestforschung mache mit den Sicherheitsbehörden gemeinsame Sache, schließt an mehr oder weniger gut begründete ältere Vorwürfe eines Teils der radikalen Linken an (Mohr/Rübner 2010). Die Ablehnung bekommen die Forscher*innen in Göttingen am deutlichsten zu spüren, aber die Skepsis strahlt auch auf andere Kolleg*innen und ihre Forschungsprojekte ab.

\section{Herausforderungen für die Protest- forschung}

Es gibt für die Protestforschung drei gewichtige Herausforderungen durch die politisch beauftragte Forschung: Erstens ist eine so finanzierte Forschung institutionell zumindest teilweise an den Sicherheitssektor bzw. an die volatilen Geldflüsse der Förderprogramme des Familienministeriums gebunden. Sie sind damit auch Teil eines sich schon mindestens 
seit Beginn des Jahrtausends abzeichnenden Prozesses der Pluralisierung der Akteurslandschaft, in der verschiedene außerwissenschaftliche Träger Monitoring und teilweise Forschungsaufgaben übernehmen (institutionelle Ebene). Dies verstärkt, zweitens, eine Tendenz in der Protestforschung, die diese ohnehin von ihren grundlagenorientierten „Mutterwissenschaften“, vor allem der theoriefokussierten Soziologie, unterscheidet: Es geht ihr stärker um konkrete Fälle (also einzelne Bewegungen oder Mobilisierungen), von denen das Interesse der Forschenden oft seinen Ausgang nimmt.

Bisher hat der relativ begrenzte theoretische Kanon der Protestforschung (dominiert von den Ansätzen Ressourcenmobilisierung, Gelegenheitsstrukturen, Framing, kollektive Identität) für eine gewisse wissenschaftlich-fachliche Identität gesorgt. Diese ist außerdem verbunden mit einem dichten, auch stark personal geprägten Netzwerk von Forschenden (Rucht 2011) und orientiert sich inhaltlich überwiegend an Prozessen und Faktoren, die eine Erklärungskraft für Aufkommen und Erfolg oder Misserfolg sozialer Bewegungen haben - und zwar weitgehend unabhängig von deren politischer oder religiöser Färbung. ${ }^{9}$

Politische Aufträge des Monitorings und der Prävention indes folgen einer anderen Logik. Solche Forschung ist angehalten oder zumindest verleitet, die wenig tragfähigen Kategorien und Wahrnehmungsmuster des Extremismusparadigmas zu übernehmen und sei es nur als förderstrategisch nützliche Referenz (inhaltliche Ebene). Drittens gefährdet eine politisch beauftragte Forschung, wie oben dargestellt, den Zugang zum eigenen Forschungsfeld (relationale Ebene).

Diese Problemstellungen existieren unabhängig von den Intentionen der konkreten Forschenden. Sie entstehen als Folge struktureller Kopplungen und ihrer Wahrnehmung. Der außerwissenschaftliche Einfluss verdankt sich teilweise (bspw. im Fall von Mittelzuweisung und -entzug) direkter staatlicher Sanktionsmacht, ist ansonsten aber vor allem subtileren Charakters. ${ }^{10}$ Wie mit den Herausforderungen umgegangen wird, ist ein wesentlicher Faktor dafür, inwiefern eine eingreifende Forschung in öffentlicher Verantwortung gelingt. Die Reaktionen der Göttinger Forschungsstelle auf Kritik war bislang von einer Haltung der Selbstverteidigung geprägt. ${ }^{11}$ Die apodiktische Behauptung der Unabhängigkeit kann aber angesichts der komplexen Situation nicht zufriedenstellen. Sie ist aus soziologischer, insbesondere machtanalytischer Perspektive kaum haltbar. Wenn sich aber der Eindruck verstärkt, dass die Auseinandersetzung mit ernstzunehmender Kritik und den eigenen Verstrickungen zu kurz kommt, ${ }^{12}$ wird Vertrauen in großen Teilen des Fachs und des Forschungsfeldes aufs Spiel gesetzt.

Es wäre jedoch falsch, nur mit dem Finger auf die Kolleg*innen in Göttingen zu zeigen. Denn der aktuelle Anlass verdeutlicht nur die eingangs eingeführte Grundspannung zwischen den gesellschaftlichen Feldern, deren Logiken sich in der Protestforschung überlagern und gegenseitig überformen. Das gilt insbesondere dann, wenn die wissenschaftliche Forschung einen expliziten politischen Auftrag hat. Der Fokus der Göttinger Bundesfachstelle auf die radikale Linke findet seine Entsprechung in der Konzentration auf die extreme Rechte in Potsdam und Jena. Es ist zu mutmaßen, dass letztere sowohl aus dem linken Feld als auch aus der Forschungslandschaft lediglich deshalb weniger kritisch kommentiert wurden, weil dort eine grundlegende politische Affinität zu deren Aufgabenstellung vorherrscht.

Kein"e Protestforscher"in forscht „wertfrei“. Auch die „normale“ Forschung an Universitäten ist selbstverständlich in Abhängigkeitsgefüge verstrickt. Aber Abhängigkeiten von auf Erfolg getrimmten Institutsleitungen, von fachpolitischen Orientierungen folgenden DFG-Kollegien oder themenkonjunktur-getriebenen BMBF-Ausschreibungen sind im Feld außerparlamentarischer Politik weniger politisch aufgeladen; die Betreffenden stehen daher weniger unter Rechtfertigungsdruck. Der Blick auf die behandelten Einrichtungen wirft also ein Schlaglicht auf uns alle. 


\section{Der Protestforschung ins Stammbuch geschrieben}

Der Fall der Protestforschung mit politischem Auftrag wirft noch weitere Fragen auf. In den Blick kommen beispielsweise die Bedingungen wissenschaftlicher Forschung über Proteste und Bewegungen im „akademischen Kapitalismus“ im Zeichen von Projektförmigkeit, zunehmender Drittmittelabhängigkeit und sinkenden Förderquoten. Diese Entwicklungen werden die Verlockungen von Finanzquellen außerhalb der klassischen Wissenschaftsförderung nicht kleiner werden lassen.

Auch die Frage nach dem Umgang mit Medien und Öffentlichkeit stellt sich in diesem Zusammenhang eindringlich. Publizität und öffentliche Wirksamkeit sind nicht nur ein Ideal einer engagierten „Public Sociology“, sondern immer mehr auch ein unhintergehbarer Imperativ für Wissenschaftler*innen und wissenschaftliche Einrichtungen, die sich unter Bedingungen stetig wachsenden Konkurrenzdrucks behaupten wollen. Auch diese Entwicklung setzt eher Anreize, für schnelle Erfolge in der Aufmerksamkeitsökonomie Abstriche bei der wissenschaftlichen Strenge zu machen. Was ist also zu tun, wenn die Antwort auf diese Herausforderung nicht im sich selbst bloßstellenden Rückzug auf das reine, scheinbar interesselose Interesse an Erkenntnis liegt?

Protestforscher*innen werden weiterhin unter besonderer Beobachtung stehen. Sie können sich angesichts der widersprüchlichen gesellschaftlichen Erwartungen nicht nicht positionieren. Dieser Druck sollte zuallererst als Aufforderung zu einem offensiven Umgang mit der eigenen Position verstanden werden. Das Gebot der Transparenz bezieht sich auf den institutionellen und politischen Sprechort, auf die Finanzierung, auf die Kooperationen und den Verwertungskontext. Insbesondere dann, wenn die Forschung im politischen Auftrag stattfindet, muss dieser klar formuliert sein. Diese Transparenz ist nicht nur ein forschungsethischer Grundsatz. Sie ist auch von praktischem Nutzen, denn sie stellt einen Maßstab zur kritischen Bewertung der Forschung, auch für Außenstehende, bereit.
Die zweite Forderung ist die nach Stärkung der theoretischen und methodologischen Debatten in der Protestforschung. Diese sollten vom aufrichtigen Interesse an der Analyse der Dynamiken und Entwicklungen unseres Gegenstandes im breitesten Sinne, also auch unabhängig von politischen Präferenzen, geleitet sein. Dies lässt für politische Schlussfolgerungen noch genügend Raum - in Ergänzung zur Forschung. Zur Diskussion der Forschung mit politischem Auftrag gehört wesentlich, die Zielstellungen selbst zur Diskussion zu stellen. Welche Entwicklungen in sozialen Bewegungen werden für eine an Demokratie und Menschenrechten orientierte Gesellschaft als problematisch angesehen? Mit welchem Verständnis von Demokratie und Menschenrechten?

Protestforschung muss sich drittens, um die ersten beiden Forderungen überhaupt umsetzen zu können, einem Metadiskurs aussetzen und in sich schnell wandelnden Kontexten immer wieder neu ihre Rolle im Gefüge der Erwartungen ausbuchstabieren. Angezeigt wäre daher auch eine wissenschaftssoziologische Erforschung der Protestforschung, also eine systematische Beobachtung der Beobachter"innen von Protest und eine Bilanzierung der empirischen Erträge unterschiedlicher institutioneller Kontexte.

Ein Szenario, das es bei aller gegenseitigen Kritik zu vermeiden gilt, ist eine Frontstellung zwischen unterschiedlichen Gruppen oder Institutionen, die sich dem Primat der Wissenschaftlichkeit im akademischen Diskurs über einen gemeinsamen Forschungsgegenstand verbunden fühlen. Es gibt einige Disziplinen, in denen die politische Überlagerung der Fachdebatte zu einer aggressiven Diskussionskultur und zu einer starken Polarisierung beigetragen hat, bis hin zu Angriffen auf die persönliche Integrität. Die Gender-Studies sind ein Beispiel für gezielte Angriffe vor allem von außen. Interne Friktionen gibt es in der Forschung zu Rassismus, Migration und Integration.

Insbesondere die Antisemitismusforschung ist ein Beispiel für eine sich in den letzten Jahren zuspitzende Polarisierung zwischen kaum noch kommunikationsfähigen Lagern mit 
abgegrenzten Zitationsnetzwerken. Debatten werden zunehmend in Ethikgremien oder vor Gericht geführt, statt auf Konferenzen (vgl. Ullrich/Kohlstruck 2017). Der Hintergrund ist eine Überdeterminierung durch einen politischen Diskurs über Haltungen zum Nahostkonflikt. Wissenschaftliche Inhalte und Kommunikationsformen haben nur noch begrenzte Strukturierungskraft. Um ein solches Szenario in der sich pluralisierenden Protestforschung zu verhindern, braucht es mehr denn je einen kontinuierlichen, institutionsübergreifenden, (selbst-) kritischen Dialog über die Aufgaben des Forschungsfelds, seine Fragen, Methoden und Indienstnahmen.

Simon Teune und Peter Ullrich sind Mitglieder des Instituts für Protest- und Bewegungsforschung (ipb), Teune ist zudem Vorsitzender des ipb-Vorstands. Gemeinsam leiten sie den Forschungsbereich „Soziale Bewegungen, Technik, Konflikte“ am Zentrum Technik und Gesellschaft der TU Berlin.

Kontakt: teune@ztg.tu-berlin.de / ullrich@ ztg.tu-berlin.de

\section{Anmerkungen}

${ }^{1}$ So sinngemäß ein konservativer Politiker in einer Gremiendebatte über die Einrichtung des Instituts für Protest- und Bewegungsforschung (ipb).

2 Ob Forschung zu Protesten auch in dem bald entstehenden „Institut für gesellschaftlichen Zusammenhalt" eine Rolle spielen wird, bleibt abzuwarten. Die millionenschwere Förderinitiative des BMBF ist auch als Reaktion auf völkische Proteste zu verstehen und hat einen expliziten Auftrag zur Intervention.

${ }^{3}$ http://www.spd-fraktion-niedersachsen.de/ imperia/md/content/ltf/koalitionsvereinbarung_rot-gr__n_20130214.pdf\#page=16 (abgerufen am 26. Februar 2018)

${ }^{4}$ http://www.mi.niedersachsen.de/ aktuelles/presse_informationen/dokumentations--und-forschungsstelle-begleitet-arbeit-des-niedersaechsischen-verfassungsschutzes--pistorius-weiterer-wichti- ger-schritt-beim-kampf-gegen-extremismus-in-unserem-land--148456.html (abgerufen am 26. Februar 2018)

5 Der Begriff „linke Militanz“ ist aus der Diskussion um die Ausrichtung des Bundesprogramms ,Initiative Demokratie stärken hervorgegangen, das Projekte unterstützen sollte, die „präventiv gegen Linksextremismus und islamistischen Extremismus" vorgehen. In der Evaluation des Programms wurde deutlich, dass der Begriff des „Linksextremismus" nicht geeignet war, um eine Zielgruppe zu definieren, und dass pädagogische Maßnahmen, die auf diesem Konzept aufbauten, ins Leere liefen.

${ }^{6} \mathrm{Zu}$ beiden Stellen fehlt es (Stand: März 2018) an Informationen auf der Webseite des Instituts für Demokratieforschung. Hinweise finden sich bis zum ersten Demokratie-Dialog ausschließlich beiläufig in Stellenausschreibungen und indirekt in wenigen Blogeinträgen. Auch die Profilseiten der Mitarbeiter"innen lassen keine Rückschlüsse darauf zu, wer den Einrichtungen zugerechnet werden kann.

${ }^{7}$ Frühe Kritik regte sich im AStA der Universität Göttingen: https://asta. uni-goettingen.de/wp-content/uploads/2017/02/2017_02_17-Verfassungschutz-und-Wissenschaft.pdf (aufgerufen am 26.2.2018).

${ }^{8}$ Aus dem Flugblatt zur Aktion auf Indymedia: https://de.indymedia.org/node/15106 (aufgerufen am 26.2.2018).

9 Dass dies trotzdem schon immer ein Spannungsverhältnis darstellt, zeigt sich daran, dass de facto ein Großteil der empirischen Forschungen und der Theoriearbeit sich doch an progressiven Bewegungen abgearbeitet hat. Aber es ist längst Konsens, dass der Bewegungsbegriff trotz seiner auch hegelianisch-marxistischen Prägungen nicht für diese reserviert sein kann. Soziale Bewegungen als Form sind in der "Bewegungsgesellschaft” (Neidhardt/Rucht 1993) ubiquitär.

${ }^{10}$ Man denke beispielsweise an die Verlockungen, die mit der Einrichtung für FoDEx zur Verfügung stehenden Textkorpora des 
Niedersächsischen Verfassungsschutzes als Daten zu verwenden, auch wenn sie nach nicht-wissenschaftlichen bzw. nicht überprüfbaren Kriterien zustande gekommen sind.

${ }^{11}$ Ablesbar ist diese Haltung u. a. in einer Erklärung des Göttinger Instituts zur Schnipsel-Aktion (http://www.demokratie-goettingen. de/aktuelles/schnipsel-aktion-vor-dem-institut-am-11-11-2017) oder im Vorwort des ersten Demokratie-Dialogs: „Völlig unzweifelhaft ist dabei, dass die Wissenschaftler und Archivare der Forschungs- und Dokumentationsstelle in ihrer Arbeit frei und unabhängig sind. Jedwede Einflussnahme, etwa durch den Verfassungsschutz, aber auch durch das Innenministerium oder andere Regierungsinstitutionen und Behörden, ist ausgeschlossen" (Trittel et al.: 3).

${ }^{12}$ Die Reaktion auf Kritik an der Forschungsstelle erinnert an die Auseinandersetzung um die Studie „Die neue Macht der Bürger“ (Marg et al. 2013) des Göttinger Instituts. Auch damals führte die in Teilen überzogene, aber durchaus berechtigte Kritik an mangelnder Transparenz über die Finanzierung durch BP gegenüber Interviewpartner*innen zu einer Abwehrhaltung im Feld.

\section{Literatur}

Ackermann, Jan/Behne, Katharina/ Buchta, Felix/Drobot, Marc/Knopp, Philipp 2015: Metamorphosen des Extremismusbegriffes. Diskursanalytische Untersuchungen zur Dynamik einer funktionalen Unzulänglichkeit. Wiesbaden: Springer

Daphi, Priska/Kocyba, Piotr/Roose, Jochen/Rucht, Dieter/Zajak, Sabrina/Neuber, Michael/Scholl, Franziska/Sommer, Moritz/ Stuppert, Wolfgang 2015: Protestforschung am Limit. Eine soziologische Annäherung an Pegida. ipb working papers. Berlin. https://protestinstitut.eu/wp-content/uploads/2015/03/ protestforschung-am-limit_ipb-working-paper_web.pdf [16.3.2018]

Forum für Kritische Rechtsextremismusforschung (Hg.) 2011: Ordnung. Macht.
Extremismus: Effekte und Alternativen des Extremismus-Modells. Wiesbaden: VS Verlag. Marg, Stine/Geiges, Lars/Butzlaff, Felix/ Walter, Franz (Hg.) 2013: Die neue Macht der Bürger. Was motiviert die Protestbewegungen? Reinbek bei Hamburg: Rowohlt.

Mohr, Markus/Rübner, Hartmut 2010: Gegnerbestimmung. Sozialwissenschaft im Dienst der inneren Sicherheit. Münster: Unrast.

Neidhardt, Friedhelm/Rucht, Dieter 1993: Auf dem Weg in die „Bewegungsgesellschaft“? Über die Stabilisierbarkeit sozialer Bewegungen. In: Soziale Welt, Jg. 44, 305-326.

Reuband, Karl-Heinz 2015: Wer demonstriert in Dresden für Pegida? Ergebnisse empirischer Studien, methodische Grundlagen und offene Fragen. In: Mitteilungen des Instituts für Parteienrecht und Parteienforschung, Nr. 21, 133-43.

Riedl, Jonathan/Micus, Matthias 2017: Der blinde Fleck des Extremismus(-Begriffes). Überlegungen zu einer möglichen Alternative. In: Demokratie-Dialog, Jg. 1, Heft 1, 16-22.

Quendt, Matthias 2017: Vorurteilsgeleitete Radikalisierung als integratives Konzept öffentlicher Demokratieforschung. In: Wissen schafft Demokratie, Nr. 1, 104-123.

Trittel, Katharina/Micus, Matthias/Marg, Stine/Geiges, Lars 2017. Demokratie-Dialog. Die Arbeit des Instituts für Demokratieforschung im Rahmen der „Forschungs- und Dokumentationsstelle zur Analyse politischer und religiöser Extremismen in Niedersachsen ". In: Demokratie-Dialog, Jg. 1, Heft 1, 2-9.

Teune, Simon/Ullrich, Peter 2015: Demonstrationsbefragungen - Grenzen einer Forschungsmethode. In: Forschungsjournal Soziale Bewegungen, Jg. 28, Heft 3, 95-100.

Ullich, Peter/Kohlstruck, Michael 2017: Muster der öffentlichen Kommunikation über Antisemitismus. Das Beispiel der Rezeption der Studie „Antisemitismus als Problem und Symbol“. conflict \& communication online Jg. 16, Heft 1. 


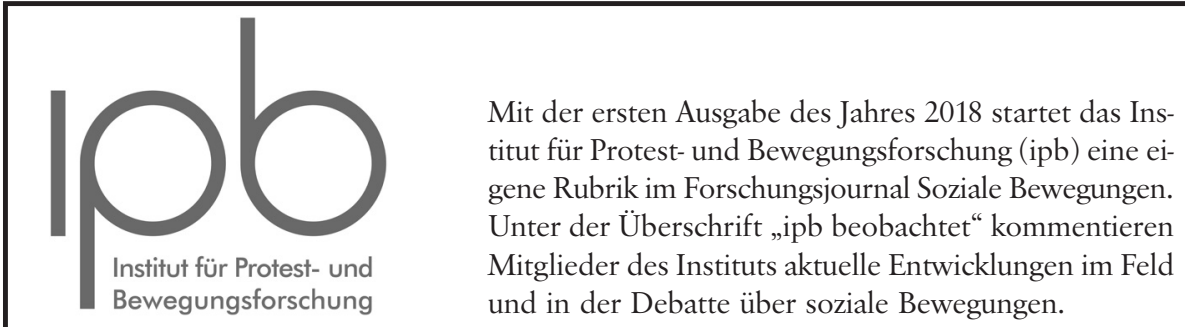

Das ipb ist ein selbstorganisiertes Netzwerkinstitut, das zurzeit 115 Forschende an Universitäten, Fachhochschulen und außeruniversitären Forschungseinrichtungen vor allem im deutschsprachigen Raum zusammenbringt, um sich über das Forschungsfeld auszutauschen und eigene Forschungsprojekte anzustoßen. Jede*r, der/die in dem Themenfeld forscht oder die Arbeit des ipb unterstützen will, kann Mitglied, bzw. Fördermitglied werden.

In der Infobox, die die Rubrik begleitet, berichtet das ipb aus der laufenden Arbeit.

Im Frühjahr 2018 hat das ipb eine Spendenkampagne gestartet, um grundlegende Koordinationsarbeiten dauerhaft finanzieren zu können. Der überwiegende Teil der Aktivitäten des ipb baut auf unbezahlter Arbeit auf. Der Koordinationsaufwand, der mit der Vernetzung von Kolleg*innen und der Initiierung von Forschungsprojekten einhergeht, muss aber bezahlt werden. Dazu reicht das Aufkommen aus Mitgliedsbeiträgen bislang nicht aus. Spenden kann man über die ipb-Webseite unter https://protestinstitut.eu/spenden/. Spenden an den Verein, der das Institut trägt, sind von der Steuer absetzbar.

$\mathrm{Zu}$ Problemen und Herausforderungen im Feld der Protest- und Bewegungsforschung finden in loser Folge auch Diskussionen im Rahmen des 14-tägigen Kolloquiums „Politik von unten" statt. In der Vergangenheit ging es in diesen Metadebatten unter anderem um die Probleme der Drittmittelfinanzierung, um das Verhältnis von Forschenden und Aktivist*innen und Protestforschung im Kontext des Extremismusparadigmas. Hauptsächlich dient das Kolloquium jedoch zur Diskussion von unveröffentlichten Texten. Das aktuelle Programm findet sich unter https://protestinstitut.eu/veranstaltungen_uberblick/\#kolloquium

Bei mehreren herausragenden Protesten hat das ipb in der Vergangenheit Demonstrationsbefragungen durchgeführt. Die Befragung von Teilnehmenden bei Demonstrationen ist eine wichtige Methode, um die Zusammensetzung der Protestierenden und ihre Sicht in einem Konflikt abzubilden. Das ipb hat Befragungen bei Pegida, bei den Mahnwachen für den Frieden („Montagsmahnwachen“), bei den Protesten gegen TTIP/CETA und gegen den G20-Gipfel in Hamburg durchgeführt. Ergebnisse der Befragungen waren im Forschungsjournal und auf der ipb-Webseite zu finden.

Ein aktuelles Beispiel für die vernetzte Forschung des ipb ist das Projekt „Mapping \#NoG20. Dokumentation und Analyse der Gewaltdynamik im Kontext der Proteste gegen den G20-Gipfel in Hamburg 2017“. In dem Projekt arbeiten mehr als 20 Forscher“innen aus unterschiedlichen Perspektiven an einer Rekonstruktion der Eskalation der Gewalt. Die Gruppe fragt, wie in konkreten Interaktionen grundlegende Rahmenbedingungen zum Tragen kommen und wie der Konflikt von verschiedenen Akteuren gedeutet wird. Informationen zum Projekt finden sich unter: https://protestinstitut.eu/projekte/mapping-nog20/

Informationen über die laufenden Aktivitäten verbreitet das ipb auch über Facebook: https://www.facebook.com/Protestinstitut und Twitter: https://twitter.com/protestinstitut 